\title{
CONHECIMENTO DOS ACADÊMICOS DE ENFERMAGEM ACERCA DA HEPATITE C
}

\section{KNOWLEDGE OF NURSING ACADEMICS ABOUT HEPATITIS C}

\section{CONOCIMIENTO DE LOS ACADÉMICOS DE ENFERMERÍA ACERCA DE LA HEPATITIS C}

Annanda Luyza Carias Maia ${ }^{1}$, Cristiane da Câmara Marques ${ }^{2}$, Bárbara Coeli Oliveira da Silva ${ }^{3}$, Vanessa Pinheiro Barreto ${ }^{4}$, Walida Andreza de Oliveira Gomes ${ }^{5}$, Alexsandra Rodrigues Feijão ${ }^{6}$

\begin{abstract}
RESUMO
Objetivo: avaliar o conhecimento de acadêmicos de enfermagem acerca da hepatite C. Método: estudo transversal desenvolvido em uma universidade pública do nordeste brasileiro. Participaram da pesquisa 129 graduandos matriculados entre o $5^{\circ}$ e $9^{\circ}$ períodos. A obtenção dos dados ocorreu por questionário auto-aplicado entre setembro e novembro de 2015 . O instrumento aplicado aos estudantes foi constituído em duas partes: 1) sete perguntas relacionadas a dados pessoais para compor a caracterização dos sujeitos; 2) nove questões de múltipla escolha, específicas sobre hepatite $\mathrm{C}$ indagando sobre prevenção, transmissão, medidas pós-exposição, sinais e sintomas, complicações e tratamento. Resultados: a maioria dos participantes apresentou nível de conhecimento moderado $(69,7 \%)$, seguido de baixo nível $(21,7 \%)$. Verificou-se a associação de alto nível de conhecimento com semestre avançado do curso ( $\mathrm{p}$ 0,05), o que não foi observado nas demais variáveis como outra formação ou participação em projetos relacionados ao tema. Conclusão: o conhecimento acerca da hepatite $\mathrm{C}$ é reduzido entre estudantes de enfermagem, portanto sugerem-se estratégias de ensino que viabilizem maior aproximação com temática tão relevante na formação do enfermeiro.

Descritores: Hepatite C; Hepatite Viral Humana; Estudantes de Enfermagem; Conhecimento; Enfermagem.
\end{abstract}

\footnotetext{
${ }^{1}$ Enfermeira. Especialista em Gestão Hospitalar e Serviços de Saúde. Enfermeira da Secretaria de Estado da Saúde Pública do Rio Grande do Norte.

${ }^{2}$ Enfermeira. Mestra em Enfermagem. Enfermeira da Clínica Integral Saúde.

${ }^{3}$ Enfermeira. Doutoranda em Enfermagem da Universidade Federal do Rio Grande do Norte. Enfermeira da Secretaria de Estado da Saúde Pública do Rio Grande do Norte e Secretaria Municipal de Saúde de Parnamirim.

${ }^{4}$ Enfermeira. Doutoranda em Enfermagem da Universidade Federal do Rio Grande do Norte.

${ }^{5}$ Enfermeira. Especialista em Gestão Hospitalar. Enfermeira da Prontoclínica Doutor Paulo Gurgel.

${ }^{6}$ Enfermeira. Doutora em Enfermagem. Docente do Departamento de Enfermagem e do Programa de PósGraduação em Enfermagem da Universidade Federal do Rio Grande do Norte.
} 


\begin{abstract}
Objective: to evaluate the knowledge of nursing students about hepatitis C. Method: a crosssectional study developed at a public university in the Brazilian Northeast. 129 students enrolled in the 5th to 9th semester participated in the study. Data were obtained by self-administered questionnaire between September and November 2015. The instrument applied to students consisted of two parts: 1) seven questions related to personal data to compose the characterization of the subjects; 2) Nine hepatitis $\mathrm{C}$ specific multiple choice questions asking about prevention, transmission, post-exposure measures, signs and symptoms, complications and treatment. Results: the majority of the participants had a moderate level of knowledge (69.7\%), followed by a low level $(21.7 \%)$. The association of high level of knowledge with advanced semester of the course was verified ( $\mathrm{p}$ 0.05), which was not observed in the other variables as another formation or participation in projects related to the theme. Conclusion: the knowledge about hepatitis $\mathrm{C}$ is reduced among nursing students, so teaching strategies are suggested that allow greater approximation with such a relevant topic in nurses' education.

Descriptors: Hepatitis C; Hepatitis Viral Human; Students Nursing; Nursing.
\end{abstract}

\title{
RESUMEN
}

Objetivo: evaluar el conocimiento de académicos de enfermería acerca de la hepatitis $\mathrm{C}$. Método: estudio transversal desarrollado en una universidad pública del nordeste brasileño. Participaron de la investigación 129 graduandos matriculados entre el $5^{\circ}$ y $9^{\circ}$ períodos. La obtención de los datos ocurrió por cuestionario auto-aplicado entre septiembre y noviembre de 2015. El instrumento aplicado a los estudiantes constaba de dos partes: 1) siete preguntas relacionadas con datos personales para componer la caracterización de las asignaturas; 2) Nueve preguntas de opción múltiple específicas para la hepatitis $\mathrm{C}$ que se refieren a la prevención, transmisión, medidas posteriores a la exposición, signos y síntomas, complicaciones y tratamento. Resultados: la mayoría de los participantes presentó nivel de conocimiento moderado $(69,7 \%)$, seguido de bajo nivel $(21,7 \%)$. Se verificó la asociación de alto nivel de conocimiento con semestre avanzado del curso (p 0,05$)$, lo que no fue observado en las demás variables como otra formación o participación en proyectos relacionados al tema. Conclusión: el conocimiento acerca de la hepatitis $\mathrm{C}$ es reducido entre estudiantes de enfermería, por lo que se sugieren estrategias de enseñanza que viabilicen mayor aproximación con temática tan relevante en la formación del enfermero.

Descriptores: Hepatitis C; Hepatitis Viral Humana; Estudiantes de Enfermería; Enfermería.

\section{INTRODUÇÃO}

A hepatite $\mathrm{C}$ é uma doença inflamatória hepática, cujo agente etiológico é o vírus da hepatite C (HCV). Atualmente revela-se como grave problema de saúde pública no Brasil e no mundo por ser responsável direto de casos de cirrose, competindo com a hepatite alcoólica, como principal causa de hepatopatia crônica. ${ }^{1-2}$

Embora haja disseminação mundial e gravidade clínica, a hepatite $\mathrm{C}$ ainda é conhecida como uma epidemia chamada "silenciosa". De acordo com o boletim epidemiológico de Hepatites Virais de 2015, foram notificados mais de 17.000 
casos no Brasil de hepatite $\mathrm{C}$ no Sistema de Informação de Agravos Notificados ano de 2014, considerado um dado preocupante..$^{3-4}$ As estimativas mostram que cerca de $20 \%$ dos infectados cronicamente pelo $\mathrm{HCV}$ podem evoluir para cirrose hepática, e de $1 \%$ a $5 \%$ para câncer de fígado. Assim, no ocidente, o HCV é uma das principais indicações de transplante hepático e causa relevante de morbimortalidade no mundo. ${ }^{5}$

Vale ressaltar que a hepatite $\mathrm{C}$ envolve inúmeras formas de transmissão. Dentre elas destaca-se a exposição ocupacional ao HCV, uma vez que a infecção subclínica prevalece e a maioria dos pacientes não tem sintomatologia na fase aguda, deixando os profissionais de saúde, principalmente, os da enfermagem, mais vulneráveis. Tal fato gera maior necessidade de conhecimento por parte desses profissionais. ${ }^{6}$

O conhecimento sobre a natureza, sintomas, transmissão, prevenção e tratamento da hepatite $\mathrm{C}$ são elementos chaves para acadêmicos de enfermagem, pois como integrantes do sistema de produção em saúde, seja na assistência de enfermagem ou produção de conhecimento em pesquisa, podem atender pessoas infectadas pelo $\mathrm{HCV}^{7}$

Como futuros enfermeiros, profissional de saúde que passa a maior parte do dia ao lado dos pacientes, o discente precisa ser preparado durante sua formação acadêmica para que consiga realizar os cuidados clínicos adequados, além de estar ciente dos equipamentos individuais de segurança corretos a serem utilizados. ${ }^{8}$

Assim, percebe-se a necessidade de os estudantes de enfermagem receberem capacitação visando promoção, prevenção e tratamento clínico adequados ao $\mathrm{HCV}$, ou seja, terem conhecimentos suficientes, o que culmina na necessidade da abordagem do tema com maior tempo e profundidade ao longo do curso de graduação. ${ }^{9}$

Diante desse contexto, este estudo tem o objetivo avaliar o conhecimento dos acadêmicos de enfermagem acerca da hepatite C. Por se tratar de uma temática de relevância para a formação do enfermeiro, espera-se que esta pesquisa possibilite a implementação de ações junto aos estudantes, contribuindo para sua formação acadêmica, bem como para novas pesquisas científicas.

\section{MÉTODO}

Estudo do tipo transversal, descritivo, de abordagem quantitativa, desenvolvido em uma universidade pública do nordeste brasileiro. A população do estudo foi composta pelos 211 alunos do curso de graduação em enfermagem que estavam matriculados entre os $5^{\circ}$ e $9^{\circ}$ períodos no 
segundo semestre de 2015. Dentre estes, 42 alunos eram do $5^{\circ}$ período, 46 alunos do $6^{\circ}$ período, 39 alunos do $7^{\circ}$ do período, 38 alunos do $8^{\circ}$ período e 46 alunos do $9^{\circ}$ período. Para determinar a amostra utilizouse a fórmula de cálculo amostral para populações finitas com erro amostral de
5\%, a qual totalizou 129 alunos, que posteriormente foi estratificada por semestre, conforme demonstra a tabela 1 . Os alunos foram selecionados entre o $5^{\circ}$ e $9^{\circ}$ período em virtude de terem cursado disciplinas com conteúdo sobre hepatite C.

Tabela 1 - Distribuição da amostra por períodos dos acadêmicos de enfermagem de uma universidade pública do nordeste brasileiro.

\begin{tabular}{cccc}
\hline Estratos (períodos) & População & $\begin{array}{c}\text { \% amostra de } \\
\text { alunos por } \\
\text { período }\end{array}$ & Amostra \\
\hline $\mathbf{5}^{\mathbf{0}}$ período & 42 & $20,15 \%$ & 26 \\
采 período & 46 & $21,71 \%$ & 28 \\
$\mathbf{7}^{\mathbf{0}}$ período & 39 & $18,60 \%$ & 24 \\
$\mathbf{8}^{\mathbf{0}}$ período & 38 & $17,83 \%$ & 23 \\
$\mathbf{9}^{\mathbf{0}}$ período & 46 & $21,71 \%$ & 28 \\
Total & 211 & $100 \%$ & 129 \\
\hline
\end{tabular}

Como critérios de inclusão elegeram-se aqueles que tinham 18 anos ou mais e matriculados regularmente a partir do $5^{\circ}$ período do curso, pois já haviam integralizado disciplinas do ciclo básico e tiveram algum contato com a temática.

A coleta de dados ocorreu entre os meses de setembro a novembro de 2015, inicialmente identificaram-se em quais dias os alunos encontravam-se na instituição. Em seguida, eram abordados em sala de aula, esclarecidos sobre pesquisa e depois convidados para participar. Mediante o aceite, assinaram o Termo de Consentimento Livre e Esclarecido.
Os questionários foram aplicados por estudantes de graduação em enfermagem integrantes do Grupo de Pesquisa em Enfermagem Baseado em Evidências previamente treinados. Foi utilizado como instrumento um questionário constituído em duas partes: 1) sete perguntas relacionadas a dados pessoais para compor a caracterização dos sujeitos, contendo dados como nome (iniciais), sexo, idade, período que o discente cursa, se possui outra formação, se participou anteriormente de curso/projeto/treinamento relacionado a temática e se considera as informações 
recebidas até o momento sobre esta doença satisfatórias; 2) nove questões de múltipla escolha, específicas sobre hepatite C indagando sobre prevenção, transmissão, medidas pós-exposição, sinais e sintomas, complicações e tratamento, baseadas na literatura disponível, nos protocolos clínicos do ministério da saúde e adaptado para este estudo, e ao final uma questão discursiva a fim de avaliar o grau de dificuldade do questionário; este último item foi embasado em dois estudos. ${ }^{10-11}$

O nível de conhecimento dos alunos sobre hepatite $\mathrm{C}$ foi avaliado conforme ao número de acertos das questões, referente à segunda parte do questionário, sendo classificados nos seguintes níveis: Baixo (03 acertos), moderado (4-6 acertos) e alto (79 acertos). Os referidos níveis de acertos foram seguidos conforme estudo publicado. $^{12}$

Para a estruturação das informações e tabulação dos dados, inicialmente, utilizou-se Microsoft Excel 2010 e as análises foram realizadas por meio do software Statistical Package for the Social Sciences versão 20.0. Para verificar a associação das variáveis foi utilizado o teste qui-quadrado, sendo considerado o nível de significância de 5\%. O presente foi submetido e aprovado pelo Comitê de Ética em Pesquisa da Universidade Federal do Rio Grande do Norte, Brasil, sob protocolo $\mathrm{n}^{\circ} \quad 1.131 .724 \quad$ e $\quad$ CAAE: 46207115.5.0000.5537. Todos os participantes assinaram as duas vias referentes ao Termo de Consentimento Livre e Esclarecido.

\section{RESULTADOS}

Quanto à caracterização dos 129 acadêmicos participantes, a maior parte era do sexo feminino $(86,0 \%)$. Com relação a faixa etária, até 23 anos $(69,77 \%)$. Quanto ao grau de período estudado, $5^{\circ}$ período $(20,15 \%), 6^{\circ}$ período $(21,71 \%), 7^{\circ}$ período $(18,60 \%), 8^{\circ}$ período $(17,83 \%)$ e $9^{\circ}$ período (21,71\%). 86,05\% não possuem outra formação, 93,80\% não possuem participação em projetos e atividades relacionadas a hepatites virais. Por último, $41,86 \%$ não estão satisfeitos com as informações recebidas sobre a doença (Tabela 2). 
Tabela 2 - Características dos estudantes de enfermagem de uma universidade pública do nordeste brasileiro.

\begin{tabular}{|c|c|c|c|}
\hline \multicolumn{2}{|c|}{ Características dos estudantes } & \multirow{2}{*}{$\begin{array}{c}\mathbf{N} \\
111\end{array}$} & \multirow{2}{*}{$\begin{array}{c}\% \\
86,05\end{array}$} \\
\hline \multirow{4}{*}{ Faixa etária } & Feminino & & \\
\hline & Masculino & 18 & 13,95 \\
\hline & Até 23 anos & 90 & 69,77 \\
\hline & Acima de 23 anos & 39 & 30,23 \\
\hline \multirow{5}{*}{ Período } & $5^{\circ}$ período & 26 & 20,15 \\
\hline & $6^{\circ}$ período & 28 & 21,71 \\
\hline & $7^{\circ}$ período & 24 & 18,60 \\
\hline & $8^{\circ}$ período & 23 & 17,83 \\
\hline & $9^{\circ}$ período & 28 & 21,71 \\
\hline \multirow{2}{*}{ Com formação anterior } & Não & 111 & 86,05 \\
\hline & Sim & 18 & 13,95 \\
\hline \multirow{2}{*}{$\begin{array}{l}\text { Participação em projetos } \\
\text { relacionados a hepatites }\end{array}$} & Não & 121 & 93,80 \\
\hline & Sim & 8 & 6,20 \\
\hline \multirow{3}{*}{$\begin{array}{c}\text { Satisfação com } \\
\text { informações recebidas } \\
\text { sobre a doença }\end{array}$} & Sim & 32 & 24,81 \\
\hline & Mais ou menos & 43 & 33,33 \\
\hline & Não & 54 & 41,86 \\
\hline \multicolumn{2}{|l|}{ Total } & 129 & 100,00 \\
\hline
\end{tabular}

Os resultados mostram que os alunos do $5^{\circ}$ período obtiverem $100,0 \%$ de acertos, referente à questão 08 a qual retratava a prevenção e transmissão do vírus HCV. Já o $8^{\circ}$ período também mostrou um desempenho de $100,0 \%$ de acertos da questão 03 relacionada às principais complicações que ocorrem nos casos mais graves de hepatite $\mathrm{C}$ crônica. Ambas as questões pertencem à segunda parte do questionário.
Em relação ao grau de dificuldade do questionário realizado, $59,6 \%$ dos participantes consideraram o questionário com grau de dificuldade moderado, seguido de difícil $(29,4 \%)$ e apenas $10,8 \%$ julgaram como fácil.

Quanto ao quantitativo de questões consideradas corretas referentes à hepatite C, a maioria $(69,7 \%)$ obteve resultado entre 4 e 6 acertos, sendo considerado neste estudo como nível moderado (Tabela 3 ). 
Tabela 3 - Distribuição do nível de conhecimento sobre hepatite C dos estudantes de enfermagem de uma universidade pública do nordeste brasileiro.

\begin{tabular}{lcc}
\hline Nível de conhecimento & N & \% \\
\hline Baixo & 28 & 21,71 \\
Moderado & 90 & 69,77 \\
Alto & 11 & 8,52 \\
\hline Total & $\mathbf{1 2 9}$ & $\mathbf{1 0 0 , 0 0}$ \\
\hline
\end{tabular}

Conforme a Tabela 4, os participaram de projetos relacionados à participantes do $8^{\circ}$ período obtiveram maior hepatite $\mathrm{C}$ obtiveram um nível de porcentagem na classificação de alto nível conhecimento moderado. Notou-se, ainda, de acertos com 26,0\%, seguidos do $5^{\circ}$ período (7,6\%). Ao relacionar a que $26,3 \%$ dos estudantes que considerou o questionário difícil, apresentou um baixo classificação de acertos das questões sobre nível de conhecimento (Tabela 4).

hepatite $\mathrm{C}$ à caracterização dos estudantes, observou-se que $50,0 \%$ dos estudantes que

Tabela 4 - Classificação de acertos das questões sobre hepatite $C$ versus o perfil dos estudantes.

\begin{tabular}{|c|c|c|c|c|c|}
\hline \multirow[b]{2}{*}{ Período } & \multicolumn{3}{|c|}{ Nível de conhecimento } & \multirow{2}{*}{$\mathbf{N}$} & \multirow{2}{*}{ Valor-p } \\
\hline & Baixo & Moderado & Alto & & \\
\hline $5^{\circ}$ & $11,54 \%(n=3)$ & $80,77 \%(\mathrm{n}=21)$ & $7,69 \%(\mathrm{n}=2)$ & $\mathrm{N}=26$ & \multirow{5}{*}{$\mathbf{0 , 0 5 0}{ }^{(1)}$} \\
\hline $6^{0}$ & $28,57 \%(\mathrm{n}=8)$ & $71,43 \%(n=20)$ & $0,00 \%(\mathrm{n}=0)$ & $\mathrm{N}=\mathbf{2 8}$ & \\
\hline $7^{\mathbf{o}}$ & $29,17 \%(\mathrm{n}=7)$ & $66,67 \%(\mathrm{n}=16)$ & $4,16 \%(\mathrm{n}=1)$ & $\mathrm{N}=\mathbf{2 4}$ & \\
\hline $8^{\circ}$ & $13,04 \%(n=3)$ & $60,87 \%(n=14)$ & $26,09 \%(n=6)$ & $\mathrm{N}=\mathbf{2 3}$ & \\
\hline $9^{\circ}$ & $25,00 \%(n=7)$ & $67,86 \%(n=19)$ & $7,14 \%(n=2)$ & $\mathrm{N}=\mathbf{2 8}$ & \\
\hline \multicolumn{6}{|c|}{ Com outra formação } \\
\hline Não & $20,72 \%(\mathrm{n}=23)$ & $71,17 \%(\mathrm{n}=79)$ & $8,11 \%(\mathrm{n}=9)$ & $\mathrm{N}=111$ & \multirow{2}{*}{$0,689^{(1)}$} \\
\hline Sim & $27,78 \%(\mathrm{n}=5)$ & $61,11 \%(n=11)$ & $11,11 \%(n=2)$ & $\mathrm{N}=18$ & \\
\hline \multicolumn{6}{|c|}{ Participação de projetos relacionados à hepatite $\mathrm{C}$} \\
\hline Não & $21,49 \%(n=26)$ & $71,07 \%(\mathrm{n}=86)$ & $7,44 \%(n=9)$ & $\mathrm{N}=121$ & \multirow{2}{*}{$0,198^{(1)}$} \\
\hline Sim & $25,00 \%(\mathrm{n}=2)$ & $50,00 \%(n=4)$ & $25,00 \%(\mathrm{n}=2)$ & $\mathbf{N}=\mathbf{8}$ & \\
\hline \multicolumn{6}{|c|}{ Satisfação com as informações recebidas sobre a hepatite $C$} \\
\hline Sim & $12,50 \%(\mathrm{n}=4)$ & $75,00 \%(\mathrm{n}=24)$ & $12,50 \%(\mathrm{n}=4)$ & $\mathbf{N}=32$ & \multirow{3}{*}{$\mathbf{0 , 3 5 3} 3^{(1)}$} \\
\hline $\begin{array}{c}\text { Mais ou } \\
\text { menos }\end{array}$ & $18,60 \%(\mathrm{n}=8)$ & $74,42 \%(n=32)$ & $6,98 \%(\mathrm{n}=3)$ & $\mathrm{N}=43$ & \\
\hline Não & $29,63 \%(\mathrm{n}=16)$ & $62,96 \%(\mathrm{n}=34)$ & $7,41 \%(\mathrm{n}=4)$ & $\mathrm{N}=54$ & \\
\hline \multicolumn{6}{|c|}{ Grau de dificuldade do questionário } \\
\hline Fácil & $14,29 \%(n=2)$ & $78,57 \%(\mathrm{n}=11)$ & $7,14 \%(\mathrm{n}=1)$ & $\mathrm{N}=14$ & \multirow{3}{*}{$\mathbf{0 , 5 8 6}{ }^{(1)}$} \\
\hline Moderado & $20,78 \%(n=16)$ & $72,73 \%(\mathrm{n}=56)$ & $6,49 \%(\mathrm{n}=5)$ & $\mathrm{N}=77$ & \\
\hline Difícil & $26,32 \%(\mathrm{n}=10)$ & $60,53 \%(n=23)$ & $13,15 \%(\mathrm{n}=5)$ & $\mathrm{N}=38$ & \\
\hline
\end{tabular}

(1) Qui-quadrado 


\section{DISCUSSÃO}

Considerando a alta incidência e morbimortalidade da hepatite $\mathrm{C}$ no mundo, bem como por ser uma das principais causas da hepatopatia crônica, é iminente que os estudantes de enfermagem, na condição de futuros profissionais da saúde, tenham conhecimentos sobre a temática. ${ }^{13}$

Neste contexto, os resultados do presente estudo demonstraram que na maioria dos períodos, os acadêmicos de enfermagem apresentaram um nível moderado de acertos. Este achado é compatível com outro estudo que sinaliza um baixo aprendizado sobre Hepatite C durante o processo de formação do aluno de enfermagem. Demostra também o déficit do ensino aprendizagem e quanto isso pode repercutir na atuação como futuros enfermeiros frente a sua prática profissional. $^{14}$

Todavia, em destaque, os resultados apresentados por o $5^{\circ}$ período e $8^{\circ}$ período do curso de enfermagem da graduação, sobressaíram com alto nível de conhecimento em relação aos $6^{\circ}, 7^{\circ}$ e $9^{\circ}$ período.

O alto nível de conhecimento demostrado do $5^{\circ}$ período pode estar relacionado às aulas ministradas sobre hepatites virais, dentre elas a hepatite $C$, na qual a matriz curricular desse período oferece. Enquanto, o do $8^{\circ}$ período pode ser pertinente aos alunos terem que retomar conteúdos vistos anteriormente sobre hepatite $\mathrm{C}$, devido às exigências do assunto quando os mesmos vão para seus campos de práticas na atenção básica.

Em relação aos demais períodos, obtiveram uma média de moderado nível de conhecimento, podendo relacionar ao esquecimento do que anteriormente já tinha sido abordado durante o curso ou pela falta de capacidade do entendimento sobre a doença.

Estudo realizado na Turquia ressalta que mesmo os alunos sendo expostos à temática de assistência ao paciente com hepatite $\mathrm{C}$, é comum que com o passar do tempo eles não recordem. Tal fato pode estar associado ao pouco contato dos alunos com usuários que possuem a infecção, uma vez que não há atividades práticas obrigatórias ou estágios de conclusão de curso desenvolvidos durante a graduação, em hospitais de doenças infectocontagiosas, sobre a doença para garantir a sua prática profissional qualificada. ${ }^{15}$

Vale salientar que todos os acadêmicos do $5^{\circ}$ período acertaram uma questão que retratava a prevenção e transmissão do vírus HCV. Esse dado 
corrobora com um estudo recente realizado com os estudantes de enfermagem, cursando o $5^{\circ}$ período de uma universidade brasileira, as quais souberam responder as questões sobre transmissão referente ao questionário aplicado a respeito da hepatite C. Com isso, evidenciou-se que os alunos possuem um bom entendimento sobre a sua transmissibilidade. ${ }^{16}$

Não obstante, o $8^{\circ}$ período também se sobressaiu em relação a pergunta do que abordava as principais complicações que ocorrem nos casos mais graves de hepatite C crônica, a cirrose hepática, todos acertando a questão. Compreende que esses acadêmicos estão mais capacitados como futuros enfermeiros a oferecer uma melhor assistência aos pacientes com fibrose mínima ou moderada, de modo a reduzir a possibilidade de chegar à cirrose hepática, por apresentarem um maior nível de conhecimento sobre a doença. ${ }^{17}$

No que se refere à participação de projetos relacionados à hepatite $\mathrm{C}$ revelou que os alunos que se incluíram em projetos de pesquisa/extensão sobre a doença tiveram um nível alto de conhecimento, comparado aos que não participaram apresentando um baixo nível de conhecimento.

Em semelhança com essa avaliação está o estudo realizado com estudantes de cursos da área de saúde da Universidade Federal de Sergipe, evidenciando que os alunos que participam de projetos de pesquisa/extensão, proporcionam maiores atitudes científicas, ratificando a importância dessas atividades extracurriculares na formação acadêmica, e melhorando o conhecimento científico na graduação de enfermagem, de tal modo suas atitudes impactarão positivamente na prática clínica desses futuros enfermeiros. ${ }^{17}$ Portanto, explica o porquê dos alunos que participaram de projetos sobre hepatite $\mathrm{C}$ apresentaram maior desempenho no questionário, quando relacionado ao seu nível de conhecimento.

Pode-se perceber também nesta pesquisa que o grau dificuldade do questionário foi um ponto a ser levado em consideração. Profere que o pouco conhecimento dos estudantes sobre determinado assunto pode estar diretamente relacionado com a dificuldade maior de ter um bom entendimento desse tema no curso de graduação, evidenciado pelo baixo número de acertos. Isso tornar-se explícito nesse estudo, de forma que os acadêmicos que exibiram um moderado a baixo nível de conhecimento foram concomitantemente os mesmos que consideraram o questionário difícil. $^{19}$ 
Contudo, os resultados do presente estudo reforçam a importância de trabalhar o conhecimento dos estudantes da graduação de enfermagem, pois alguns períodos exibiram um nível alto de conhecimento. Entretanto, a maioria apresentou um nível moderado a baixo. Trabalhar o conhecimento dos estudantes é fundamental para que possam compreender a importância do conhecimento sobre a hepatite $\mathrm{C}, \mathrm{e}$ tornar $\mathrm{o}$ conhecimento igualitário e qualificado. Pois, além de serem futuros enfermeiros preparados para assistência ao paciente com hepatite $\mathrm{C}$, são agentes multiplicadores da informação à população. $^{19}$

Frente a todos esses achados, ressalva-se que embora não tenha evidenciado significância estatística nos resultados, a análise destes foi importante para serem discutidos, com a finalidade de contribuir diretamente para a formação dos futuros profissionais de saúde.

\section{CONCLUSÃO}

O presente estudo, demostrou que a maior dos acadêmicos apresenta um nível de moderado a baixo conhecimento. Mesmo alguns alunos se destacando com altos níveis de conhecimento. Recomenda-se que os acadêmicos de enfermagem ampliem seus estudos sobre a hepatite $\mathrm{C}$, de forma que participem de cursos de capacitações, intensifiquem as informações sobre o tema e aprimorarem os conteúdos oferecidos durante a graduação. Tal fato torna-se importante, visto que como futuros enfermeiros consigam corresponder às necessidades exigidas na assistência de enfermagem com os pacientes com hepatite C.

Acredita-se ser este um assunto com grande potencial na enfermagem; portanto, sugere-se a realização de novos estudos que reforcem a importância de ampliar o conhecimento dos estudantes de enfermagem sobre hepatite $\mathrm{C}$ durante a formação acadêmica, na qual trabalhos futuros devem ser incentivados.

No tocante as limitações do estudo, pode-se mencionar a realização em apenas uma instituição de ensino superior (IES). Assim, os resultados retratam apenas a realidade de uma determinada IES.

\section{REFERÊNCIAS}

1. Kwon, Young-Chan, Ratna BR, Ranjit $R$. Hepatitis $C$ virus infection: establishment of chronicity and liver disease progression. EXCLI J.

[Internet]. 2014 [citado em 22 out 2018]; 13:977-96. Disponível em: https://www.ncbi.nlm.nih.gov/pmc/arti 
cles/PMC4464452/pdf/EXCLI-13977.pdf

2. Hanus JS, Ceretta LB, Simões PW, Tuon L. Incidence of hepatitis $C$ in Brazil. Rev Soc Bras Méd Trop. [Internet]. 2015 [citado em 22 out 2018]; 48(6):665-73. Disponível em: http://www.scielo.br/pdf/rsbmt/v48n6/ 1678-9849-rsbmt-48-06-00665.pdf

3. Nawaz A, Zaidi SF, Usmanghani K, Ahmad I. Concise review on the insight of hepatitis C. J Taibah Univ Med Sci. [Internet]. 2015 [citado em 22 out 2018]; 10(2):132-9. Disponível em: https://reader.elsevier.com/reader/sd/pii /S165836121400119X?token=D531E5 B1C32D5041F0608572DCF9F2B4292 28B0805608D394B3F23B4C08AA62 FA58E645C60D4E81CA7CC69AE40 0B0E33

4. Ministério da Saúde (Brasil), Secretaria de Vigilância em Saúde, Departamento de DST, Aids e Hepatites Virais. Boletim Epidemiológico Hepatites Virais [Internet]. 2015 [citado em 22 maio 2016]; 4(1):1-25. Disponível em: http://www.aids.gov.br/pt-br/node/90

5. Westbrook RH, Dusheiko G. Natural history of hepatitis C. J Hepatol. [Internet]. 2014 [citado em 22 out 2018]; 61:58-68. Disponível em: https://www.journal-ofhepatology.eu/article/S01688278(14)00481-4/pdf

6. Ringehan M, McKeating JA, Protzer U. Viral hepatitis and liver cancer. Phil Trans R Soc B Biol Sci. [Internet]. 2017 [citado em 22 out 2018]; 372:20160274. Disponível em: https://royalsocietypublishing.org/doi/p df/10.1098/rstb.2016.0274

7. Ataei B, Meidani M, Khosravi M, Khorvash F, Akbari M. Knowledge, attitude, and performance of medical staff of teaching health care settings about hepatitis B and C in Isfahan, Iran. Adv Biomed Res.
[Internet]. 2014 [citado em 22 mai 2016]; 3:267. Disponível em: https://www.ncbi.nlm.nih.gov/pmc/arti cles/PMC4298875/

8. Topbaş E, Terzi B, Gayrim A, BayoğluT, Tan S. The results of a hepatitis training provided for nursing students. Int J Med ResHealth Sci. [Internet]. 2017 [citado em 16 maio 2016]; 6(3):118-24. Disponível em: https://www.ijmrhs.com/medicalresearch/the-results-of-a-hepatitistraining-provided-for-nursingstudents.pdf

9. Joukar F, Mansour-Ghanaei F, Naghipour MR, Hasandokht T. Nurses' knowledge toward hepatitis B and hepatitis $\mathrm{C}$ in Guilan, Iran. Open Nurs J. [Internet]. 2017 [citado em 16 mai 2016]; 11:34-42. Disponível em: https://opennursingjournal.com/content s/volumes/V11/TONURSJ-1134/TONURSJ-11-34.pdf

10. Souza DP. Corresponsabilidade no cuidado a pessoas com hepatite $\mathrm{C}$. [dissertação]. Florianópolis : Universidade Federal de Santa Catarina; 2014.

11. Messina JP, Humphreys I, Flaxman A, Brown A, Cooke GS, Pybus OG, et al. Global distribution and prevalence of hepatitis $\mathrm{C}$ virus genotypes. Hepatology [Internet]. 2015 [citado em 17 maio 2016]; 61(1):77-87.

Disponível em: https://aasldpubs.onlinelibrary.wiley.co m/doi/epdf/10.1002/hep.27259

12. Moreira FP, Rodrigues KL. Conhecimento nutricional e suplementação alimentar por praticantes de exercícios físicos. Rev Bras Med Esporte. [Internet]. 2014 [citado em 04 dez 2019]; 20(5): 370-3. Disponível em: http://www.scielo.br/pdf/rbme/v20n5/1 517-8692-rbme-20-05-00370.pdf

13. Rossi GC, Afonso PMD, Oliveira SLG, Furlan MLS. Hepatites B e C: o 
conhecimento dos estudantes universitários da área da saúde. Rev Enferm UERJ. [Internet]. 2010 [citado em 18 maio 2016]; 18(1):38-41.

Disponível em:

https://www.yumpu.com/pt/document/ view/28698527/38-a-41-6-2009-39hepatites-b-e-cpmd-uerj

14. Yamazhan T, Durusoy R, Tasbakan MI, Tokem Y, Pullukcu H, Sipahi OR, et al. Nursing students' immunisation status and knowledge about viral hepatitis in Turkey: a multi-centre cross-sectional study. Int Nurs Rev. [Internet]. 2011 [citado em 18 maio 2016]; 58(2):181-85. Disponível em: https://onlinelibrary.wiley.com/doi/epd f/10.1111/j.1466-7657.2010.00869.x

15. Santos SB. O conhecimento dos acadêmicos de enfermagem sobre as hepatites. [trabalho de conclusão de curso]. São Paulo: Universidade Nove de Julho; 2010.

16. Marinho RT, Barreira DP. Hepatitis C, stigma and cure. World $\mathrm{J}$

Gastroenterol. [Internet]. 2013 [citado em 18 maio 2016]; 19(40):6703-9.

Disponível em:

https://www.ncbi.nlm.nih.gov/pmc/arti cles/PMC3812468/pdf/WJG-196703.pdf

17. Figueiredo WPS, Moura NPR, Tanajura DM. Ações de pesquisa e extensão e atitudes científicas de estudantes da área da saúde. Arq Ciênc Saúde. [Internet]. 2016 [citado em 18 maio 2016]; 23(1): 47-51. Disponível em: http://www.cienciasdasaude.famerp.br/ index.php/racs/article/view/197/161

18. Santos JF, Alves AP, Stabile AM. Avaliação do conhecimento dos estudantes de enfermagem sobre sepse. Rev Eletrônica Enferm. [Internet]. 2012 [citado em 18 maio 2016]; 14(4):850-6. Disponível em: https://revistas.ufg.br/fen/article/view/1 5077/13349
19. Irmak Z, Ekinci B, Akgul AF. Hepatitis $\mathrm{B}$ and $\mathrm{C}$ seropositivity among nursing students at a Turkish university. Int Nurs Rev. [Internet]. 2010 [citado em 18 maio 2016]; 57(3): 365-9.

Disponível em: https://onlinelibrary.wiley.com/doi/epd f/10.1111/j.1466-7657.2010.00804.x

RECEBIDO: 27/08/2019

APROVADO: $10 / 03 / 2020$

PUBLICADO: 07/2020 